https://doi.org/10.22319/rmcp.v12i2.5652

Artículo

\title{
Análisis de la rentabilidad apícola por estratos en Aguascalientes, México
}

José Inés Zavala Beltrán ${ }^{\text {a }}$

Marco Andrés López Santiago ${ }^{\text {** }}$

Ramón Valdivia Alcalá ${ }^{\mathrm{a}}$

Blanca Margarita Montiel Batalla ${ }^{\text {a }}$

a Universidad Autónoma Chapingo (UACh). División de Ciencias EconómicoAdministrativas, Carretera México-Texcoco Km 38.5, Texcoco, Estado de México.

${ }^{\mathrm{b}}$ UACh. Unidad Regional Universitaria de Zonas Áridas, Durango, México.

*Autor de correspondencia: marcoandres@ chapingo.uruza.edu.mx

\section{Resumen:}

La presente investigación se enfocó en analizar la estructura de costos y la rentabilidad en el proceso de producción de la apicultura. A través de técnicas de muestreo, se seleccionaron aleatoriamente 56 apicultores de un padrón de 230, mismos que se agruparon en tres estratos: productores de 20 a 50 colmenas (pequeños), 51 a 200 colmenas (medianos) y más de 200 colmenas (grandes). Se encontró que el costo económico de producción total se compone principalmente por el costo variable, con una participación promedio relativa de $55.4 \%$ en los tres estratos. El gasto en alimentación es el concepto primordial, considerando que, para sostener la colmena, el $90.0 \%$ de los apicultores alimentan con azúcar o fructosa cuando no hay floración. El costo fijo representa el $14.0 \%$ respecto al total, la mayor erogación fue en el rubro de la depreciación en maquinaria y material de campo. Los costos de oportunidad representan el $30.6 \%$ en promedio para los tres estratos. El rendimiento promedio por colmena fue de $25.4 \mathrm{~kg} / \mathrm{año}$. En conclusión, considerando el análisis económico, para el estrato I la actividad no es viable, ya que no considera el valor de todos los recursos en el proceso productivo (costos de oportunidad), así mismo en este estrato el ingreso principal proviene de otras actividades. En términos financieros, la actividad es viable en los tres 
estratos, lo que indica que se tiene la capacidad de cubrir costos fijos y costos variables. Al incluir los costos de oportunidad, los costos fijos y variables disminuyen.

Palabras clave: Abejas, Productividad, Competitividad.

Recibido: $26 / 03 / 2020$

Aceptado: 11/09/2020

\section{Introducción}

En México, la apicultura tiene una gran importancia socioeconómica y ecológica, es considerada como una de las principales actividades pecuarias generadora de divisas ${ }^{(1)}$. Generalmente, esta actividad se asocia únicamente con la producción de miel, polen, jalea real y propóleo; sin embargo, las abejas también son fundamentales para el equilibrio del medio ambiente debido a su colaboración en la polinización. Se estima que, dentro del $90 \%$ de la polinización que ocurre en plantas con flor en todo el mundo, un $67 \%$ es llevado a cabo por insectos, constituyéndose como el grupo de polinizadores más importante, tanto para especies de plantas silvestres como cultivadas ${ }^{(2)}$.

De acuerdo con las cifras del sector ganadero, México se ubica entre el quinto y sexto lugar mundial como productor de miel, produjo 62,320 t en $2018^{(3)}$. El incremento en el valor de las exportaciones para el 2018 con respecto al año anterior fue de $15 \%^{(3)} \mathrm{y}$, en términos de volumen, $60 \%$ superior con relación a las registradas en $2017^{(1)}$.

El estado de Aguascalientes está ubicado en la región del altiplano mexicano. En esta región, debido al clima semiárido, florecen una gran cantidad de arbustos como el mezquite (Prosopis laevigata), y en época de lluvias, se presenta la floración de aceitilla (Bidens spp). En el 2018, Aguascalientes contaba con un inventario de 15,312 colmenas ${ }^{(4)}$. Con base en el Sistema Producto Apícola de Aguascalientes (2018) ${ }^{(5)}, 230$ apicultores dependen de la producción apícola con únicamente dos floraciones al año; aceitilla en noviembre y mezquite en abril. En la zona de estudio, en primavera se cosecha más del $60 \%$ de la miel, proveniente del mezquite (Prosopis laevigata) como fuente de néctar silvestre. Contrario a la región estudiada, en el sureste mexicano la gran diversidad de flores impide analizar su influencia sobre la producción de miel, bajo las condiciones agroclimáticas correspondientes ${ }^{(6)}$. Aguascalientes presenta una ventaja competitiva en la miel por ser de tipo monoflora, significa que posee características relativamente homogéneas ${ }^{(6)}$, lo que se evidencia en la diferencia del precio respecto al promedio nacional ${ }^{(7)}$. 
Sin embargo, aunque se cuenta con una ventaja competitiva, existen factores que han ocasionado que un gran número de apicultores abandonen la actividad o bajen sus niveles de producción. Un primer factor adverso lo constituye la baja precipitación, lo que repercute en una baja actividad de pecoreo y la escasez de alimento ${ }^{(8)}$. Aunado a lo anterior, se presentan bajos precios al productor en el mercado de la miel (existe varianza amplia entre el precio al productor y el consumidor final), una deficiente capacitación técnica de producción, así como un alto costo de los insumos utilizados ${ }^{(9)}$.

Otros productos importantes que produce la apicultura de esta zona son el propóleo, jalea real, polen, cera, material biológico, abeja reina y material genético ${ }^{(10)}$. En este panorama, la competitividad de todo sistema o proceso de producción en el mercado interno, se determina por su nivel de rentabilidad. La rentabilidad se estima descontando al valor de la venta de cierta cantidad de producto, los costos en los que se incurrió para obtenerlo ${ }^{(9)}$. En este sentido, es necesario realizar un análisis del costo-beneficio y calcular los precios de equilibrio en la región para los diferentes tipos de apicultores.

Por ende, el objetivo del presente trabajo fue estimar la estructura de costos, así como un análisis de flujo desembolsado, financiero y económico ${ }^{(1)}$ de la producción apícola en el estado de Aguascalientes, para determinar el nivel de utilidades unitarias o rentabilidad del sistema. La hipótesis fue que el costo del equipo, herramientas e insumos utilizados en el proceso de producción tienen una relación inversa con la rentabilidad que obtiene el apicultor en esta región.

\section{Material y métodos}

\section{Ubicación del área}

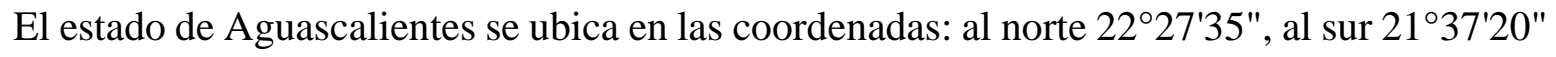
de latitud norte, al este $101^{\circ} 50^{\prime} 07^{\prime \prime}$, al oeste $102^{\circ} 52^{\prime} 27^{\prime \prime}$ de longitud oeste y colinda al norte, noreste y oeste con Zacatecas y al sureste y sur con Jalisco. Representa el $0.3 \%$ de la superficie del país ${ }^{(12)}$.

\section{Muestreo}

La muestra se calculó a partir de la población de apicultores que están adheridos al Sistema Producto Apícola del estado de Aguascalientes. Con datos del 2018, el padrón estuvo conformado por 230 apicultores $^{(5)}$. Se levantó información de campo a través de cuestionarios; estos fueron aplicados directamente a los productores de la zona de estudio. 
Los datos presentados provienen de los 11 municipios del estado de Aguascalientes. Se utilizó el criterio de estratificación aportada por Vélez ${ }^{(13)}$ y Fachini $^{(14)}$, donde se clasificó en tres categorías por el número de colmenas: Pequeños (10 a 50), Mediano (51 a 200) y Grande (más de 200). Se empleó la técnica de muestreo estratificado aleatorio. La variable que fue asociada al procedimiento de muestreo para la estimación de la varianza fue el número de colmenas por apicultor y el límite de error fue de $5 \%$.

El tamaño de la muestra final se estimó con base en la siguiente fórmula:

$$
\boldsymbol{n}=\frac{N * Z_{\alpha}^{2} * p * q}{d^{2}(N-1)+Z_{\alpha}^{2} * p * q}
$$

Donde: $\mathrm{n}=$ tamaño final de muestra; $\mathrm{Z}=1.96$ (nivel de confianza); $\mathrm{p}=$ proporción esperada (0.05); q=1-p; $\mathrm{N}=$ total de productores $(230) ; \mathrm{d}=$ precisión $(0.05)$.

La muestra se conformó por 56 apicultores incluidos en Sistema Producto Apícola estatal. Al primer estrato le correspondió el $11 \%$ (7) de la muestra final, al segundo estrato $40 \%$ (22) y al tercero $49 \%$ (27). La información se procesó mediante una hoja de cálculo de Excel.

\section{Contenido de la encuesta}

Las preguntas que incluyó el cuestionario se dividieron en los siguientes aspectos:

Manejo técnico: 1) nivel de conocimiento de los apicultores sobre las actividades productivas; 2) nivel de conocimiento técnico; 3) control del porcentaje de africanización en la zona; 4) genotipo; 5) frecuencia del cambio de la abeja reina; 6) frecuencia del cambio de colmenas; 7) alimentación de los apiarios; 8) control de enfermedades y plagas; 9) tiempo invertido en la apicultura.

Costos: 1) costos de alimentación, 2) plagas y enfermedades, 3) cambio de reina, 4) mano de obra, 5) traslado y 6) otros costos.

Producción: 1) número de productores; 2) número de colmenas; 3) producción total; 4) ubicación de las colmenas.

Ingresos: 1) precio de la miel y subproductos; 2) producción; 3) venta.

\section{Método}

Para la descripción y análisis de los aspectos de carácter social relacionados con la producción apícola, se consideraron las siguientes variables: la importancia del factor genético, el manejo técnico y el medio ambiente, la organización para la producción y asistencia técnica. Los elementos considerados para el procesamiento análisis de los coeficientes técnicos fueron: mano de obra, número de apiarios, número de colmenas, implementos alimenticios, control de enfermedades, numero de cosechas, entre otros. Se 
calcularon los costos de la mano de obra y los insumos considerando las siguientes variables: el número de jornales utilizados para las diferentes actividades, los gastos efectuados para comprar azúcar, vitaminas, control de varroa, entre otros.

La metodología de análisis de costos fue la utilizada por Sagarnaga et al ${ }^{(11)}$, donde retoma la metodología empleada por el Departamento de Agricultura de los Estados Unidos (USDA), cuya base teórica y metodológica se ajusta a los estándares recomendados por el Grupo de Trabajo sobre Costos y Retornos de la Asociación Americana de Economía Agrícola (AAEA por sus siglas en ingles).

Dentro de este contexto, el USDA clasifica los costos en dos tipos: de operación (operating costs) y generales (allocated overhead). La Universidad Estatal de Washington, retoma la clasificación de los costos en fijos y variables y los desagrega en forma de costos económicos, financieros y desembolsados. En los costos financieros se incluyen únicamente los costos fijos y variables; en los costos desembolsados se consideran, además de los costos fijos y variables, el efectivo requerido para pagar el abono a capital de los créditos de largo plazo y para cubrir el gasto familiar del productor. Los costos económicos incluyen los costos financieros y el costo de oportunidad de los factores de la producción ${ }^{(11)}$.

Se calcularon los costos de oportunidad: tierra, mano de obra, capital y gestión empresarial. Se utilizó el valor de todos los recursos en el proceso productivo, independientemente si representaban gastos desembolsados o no. Una vez cuantificados los costos de producción se procedió a determinar el precio objetivo para cada uno de los estratos, donde se identificó el precio mínimo para asegurar el cumplimiento de ganancias $^{(15)}$.

\section{Resultados}

\section{Inversiones en colmenas y equipo}

Las unidades productivas que conforman la apicultura en México están integradas por las clásicas colmenas estándares. En Aguascalientes, el apicultor conforma la colmena con su soporte (mayormente de ladrillo), piso, cámara de cría tipo langstroth y techo, con 2 alzas. La inversión por cada estrato de productores se muestra en el Cuadro 1. Respecto a la inversión de material en campo, su valor total aumenta conforme el productor aumenta de colmenas; esto debido a que necesita mayor capacidad de instalación (porta-núcleos, cámaras de cría y alzas, principalmente). El segundo y tercer estrato aumenta considerablemente en el rubro de equipo de trabajo, derivado de una mayor capacidad; algunos de los componentes necesarios son el extractor, tanque de sedimentación y banco desoperculador. Para el primer 
estrato, el $80 \%$ de los productores no cuentan con la tecnología necesaria; por lo que optan por rentar estos servicios principalmente en la cosecha.

Cuadro 1: Inversión realizada por apicultor $(\$)$

\begin{tabular}{lrrr}
\hline & \multicolumn{3}{c}{ Estratos de productores por número de colmenas } \\
& $\mathbf{1 - 5 0}$ & $\mathbf{5 1 - 2 0 0}$ & \multicolumn{1}{c}{ más de 200 } \\
\hline Equipo de trabajo & $15,805.6$ & $150,000.0$ & $165,050.0$ \\
Material en campo & $25,900.0$ & $140,535.0$ & $400,800.0$ \\
Total de inversión & $41,705.6$ & $290,535.0$ & $565,850.0$ \\
Coeficiente de variación $(\%)$ & 38.9 & 14.9 & 19.46 \\
\hline
\end{tabular}

Fuente: Elaboración propia con datos de la encuesta a apicultores, 2018.

La diferencia entre los estratos fue la inversión del equipo de campo, bodega y la calidad de dicho equipo; es decir, un extractor para 80 bastidores tiene un valor casi cinco veces mayor que el de 32 bastidores, o 10 veces más que el de lámina galvanizada. Los altos costos ocasionan que el apicultor del estrato I recurra a la renta del equipo (bodega) para la cosecha.

\section{Estructura de costos}

A partir de la información recabada de las encuestas, se estimaron los costos de producción, económico, financiero y desembolsado por estrato, de acuerdo al número de colmenas. La estructura porcentual de los costos totales está compuesta en mayor medida por los costos variables. Considerando los costos de oportunidad, el costo variable para el estrato I se compone del $51.9 \%$ (Cuadro 2), para el segundo estrato 54.1\% (Cuadro 3) y el tercer estrato $60.2 \%$ (Cuadro 4).

Cuadro 2: Estructura de costos de producción (\$) del estrato I (1-50 colmenas)

\begin{tabular}{lrrr}
\hline Concepto de costos & Económico & Financiero & Desembolsado \\
\hline Costos variables & & & \\
Alimento & $12,801.78$ & $12,801.78$ & $12,801.78$ \\
Medicamentos & 182.14 & 182.14 & 182.14 \\
Mantenimiento & $1,000.00$ & $1,000.00$ & $1,000.00$ \\
Compra de reinas & $3,295.25$ & $3,295.25$ & $3,295.25$ \\
Combustible & $6,988.89$ & $6,988.89$ & $6,988.89$ \\
Total costos variables & $24,268.07$ & $24,268.07$ & $24,268.07$ \\
Costos fijos & & & \\
Renta de terrenos & - & - & - \\
Mano de obra indirecta & - & - & - \\
Mano de obra familiar & - & - &
\end{tabular}




\begin{tabular}{lrrr}
\hline Depreciación de equipos & $2,000.00$ & $2,000.00$ & - \\
Depreciación de materia de campo & $3,000.00$ & $3,000.00$ & - \\
Otros costos fijos & $1,445.00$ & $1,445.00$ & $1,445.00$ \\
Total de costos fijos & $6,445.00$ & $6,445.00$ & $1,445.00$ \\
Costos de oportunidad & & & \\
Costo de oportunidad de la tierra & $1,500.00$ & - & - \\
(renta) & $4,170.56$ & - & - \\
Capital de trabajo & $5,600.00$ & - & - \\
Mano de obra del productor/familiar & $4,800.00$ & - & - \\
Gestión empresarial & $16,070.56$ & - & - \\
Total de costos de oportunidad & $46,783.63$ & $30,713.07$ & $25,713.07$ \\
\hline Costos totales & & & \\
\hline
\end{tabular}

Cuadro 3: Estructura de costos de producción (\$) del estrato II (51-200 colmenas)

\begin{tabular}{lrrr}
\hline Concepto de costos & Económico & Financiero & Desembolsado \\
\hline Costos variables & & & \\
Alimento & $40,319.07$ & $40,319.07$ & $40,319.07$ \\
Medicamentos & $1,008.00$ & $1,008.00$ & $1,008.00$ \\
Mantenimiento & $1,500.00$ & $1,500.00$ & $1,500.00$ \\
Compra de reinas & $14,182.08$ & $14,182.08$ & $14,182.08$ \\
Combustible & $13,640.00$ & $13,640.00$ & $13,640.00$ \\
Mano de obra & $4,008.18$ & $4,008.18$ & $4,008.18$ \\
Total de costos variables & $74,657.33$ & $74,657.33$ & $74,657.33$ \\
Costos fijos & & & \\
Renta de terrenos & $3,000.00$ & $3,000.00$ & $3,000.00$ \\
Mano de obra indirecta & - & & - \\
Mano de obra familiar & - & & - \\
Depreciación de equipos & $7,260.00$ & $7,260.00$ & - \\
Depreciación de materia de campo & $4,840.00$ & $4,840.00$ & - \\
Otros costos fijos & $2,080.00$ & $2,080.00$ & $2,080.00$ \\
Total de costos fijos & $17,180.00$ & $17,180.00$ & $5,080.00$ \\
Costos de oportunidad & & & - \\
Costo de oportunidad de la tierra (renta) & $1,500.00$ & & - \\
Capital de trabajo & $29,053.50$ & & - \\
Mano de obra del productor/familiar & $8,400.00$ & & - \\
Gestión empresarial & $7,200.00$ & & - \\
Total de costos de oportunidad & $46,153.50$ & & - \\
Costos totales & $137,990.83$ & $91,837.33$ & $79,737.33$ \\
\hline & & & - \\
\hline
\end{tabular}

Fuente: Elaboración propia con datos de la encuesta a apicultores, 2018. 
Respecto al costo fijo, se encontró que la participación es de $13.8 \%, 12.5 \%$ y $15.8 \%$ respectivamente. Siguiendo con los costos fijos, se observó que para el segundo y tercer estrato presenta una relación directa. Esto es, al tener más colmenas, el apicultor opta por adquirir tecnología de mayor capacidad. Por otro lado, se tiene la necesidad de aumentar el número de apiarios, lo que conlleva un mayor costo por renta de los terrenos.

Cuadro 4: Estructura de costos de producción (\$) del estrato III (más de 200 colmenas)

\begin{tabular}{lrrr}
\hline Concepto de costos & Económico & Financiero & Desembolsado \\
\hline Costos variables & & & \\
Alimento & $115,475.36$ & $115,475.36$ & $115,475.36$ \\
Medicamentos & $3,000.00$ & $3,000.00$ & $3,000.00$ \\
Mantenimiento & $3,600.00$ & $3,600.00$ & $3,600.00$ \\
Compra de reinas & $38,124.45$ & $38,124.45$ & $38,124.45$ \\
Combustible & $23,335.00$ & $23,335.00$ & $23,335.00$ \\
Mano de obra & $9,963.03$ & $9,963.03$ & $9,963.03$ \\
Total de costos variables & $193,497.84$ & $193,497.84$ & $193,497.84$ \\
\hline Costos fijos & & & \\
Renta de terrenos & $6,000.00$ & $6,000.00$ & $6,000.00$ \\
Mano de obra indirecta & $9,963.03$ & $9,963.03$ & $9,963.03$ \\
Mano de obra familiar & - & & - \\
Depreciación de equipos & $19,380.00$ & $19,380.00$ & - \\
Depreciación de materia de campo & $12,920.00$ & $12,920.00$ & - \\
Otros costos fijos & $2,625.00$ & $2,625.00$ & $2,625.00$ \\
Total de costos fijos & $50,888.03$ & $50,888.03$ & $18,588.03$ \\
\hline Costos de oportunidad & \multicolumn{3}{|}{} \\
Costo de oportunidad de la tierra (renta) & $3,000.00$ & & - \\
Capital de trabajo & $51,717.50$ & & - \\
Mano de obra del productor/familiar & $10,200.00$ & & - \\
Gestión empresarial & $12,000.00$ & & - \\
Total de costos de oportunidad & $76,917.50$ & & - \\
Costos totales & $321,303.37$ & $244,385.87$ & - \\
\hline
\end{tabular}
Fuente: Elaboración propia con datos de la encuesta a apicultores, 2018.

Dentro de los costos variables, el rubro con mayor participación es la alimentación, en el estado de Aguascalientes los apicultores alimentan con azúcar y fructosa. La alimentación es proporcionada cuando no hay floración y es importante para la supervivencia de la abeja; en este sentido, este costo variable tiene un crecimiento progresivo a medida que el productor aumenta el número de colmenas. El segundo rubro con mayor participación es el costo de transporte, el cual básicamente se refiere al gasto en combustible que se utiliza para la realización del manejo técnico en cada apiario. El tercer rubro corresponde a la mano de obra 
para la realización de las actividades apícolas; en temporada de cosecha (marzo-abril) se requiere el mayor número de jornales.

En cuanto a los costos fijos, se integran en mayor proporción por el valor de la depreciación de infraestructura en campo, seguido por la depreciación de los equipos de trabajo y protección. En el caso de la depreciación de los equipos de trabajo (extractor, banco desoperculador, tambo de acero inoxidable, etc.), tienen una relación inversa con el número de colmenas.

Los costos de oportunidad representan el $34.3 \%$ para el estrato I, $33.4 \%$ para el II y $24.0 \%$ para el estrato III; siendo el costo de capital de trabajo el de mayor aportación. Para el cálculo de costos de oportunidad de la mano de obra del productor, los jornales fueron cotizados en $\$ 200$ como referencia del precio de un jornal en la región. Los encuestados del primer estrato consideran que para la actividad de sus colmenas requieren 28 jornales al año, el segundo estrato considera que se usan 42 jornales y el tercer estrato 51 jornales. Los apicultores de la región consideran que la tierra tiene un costo de oportunidad de $\$ 1,500$, en el entendido de que, si estas no son usadas, se pueden rentar a otros apicultores al costo mencionado.

Para valorar la gestión empresarial, los productores del estrato I consideraron que trabajan una hora a la semana para administrar los apiarios, el estrato II 3 horas y el estrato III trabaja 5 horas a la semana para la planeación de sus actividades. El estimado del costo financiero por kilogramo de miel producido, es de $\$ 45.92$, \$31.54 y \$25.49 respectivamente para cada estrato.

\section{Ingresos y rentabilidad}

Cuando el apicultor depende mayormente de la actividad apícola, tiene más colmenas; por el contrario, cuando el apicultor posee menos colmenas, tiende a optar por dedicarse a otras actividades que le generen ingreso. Para estimar los ingresos, para el estrato I se consideró un rendimiento promedio de 19.6 kilogramos por colmena a un precio de $\$ 65$, al estrato II le correspondieron 26.4 kilogramos por colmena a un precio de $\$ 50.9$ y para el estrato III se consideraron 30.2 kilogramos por colmena a un precio de $\$ 50.45$ de acuerdo con los datos de las encuestas. 
Cuadro 5: Porcentaje de aportación al ingreso total

\begin{tabular}{llll}
\hline \multirow{2}{*}{ Concepto } & \multicolumn{3}{l}{ Estratos por número de colmenas } \\
& $\mathbf{2 0} \mathbf{5 0}$ & $\mathbf{5 1} \mathbf{~ a ~ 1 9 9}$ & $\mathbf{2 0 0}$ o más \\
\hline Aportación económica de la apicultura & 9.6 & 32.5 & 62.8 \\
miel & 97.2 & 87.0 & 88.0 \\
cera & 1.0 & 1.6 & 3.9 \\
polen & 0.0 & 0.0 & 0.3 \\
núcleos & 0.0 & 3.7 & 2.4 \\
otros & 1.8 & 7.7 & 5.5 \\
Total & 100.0 & 100.0 & 100.0 \\
\hline
\end{tabular}

Fuente: Elaboración propia con datos de la encuesta a apicultores, 2018.

A medida que el apicultor adquiere más colmenas, los ingresos generados en la actividad apícola incrementan su participación en el ingreso total (Cuadro 5). En este sentido, se pudo notar que la miel es el producto principal que se comercializa.

En términos financieros es viable en los tres estratos; es decir, se tiene la capacidad de cubrir los costos fijos y variables (Cuadro 6). Por otro lado, en términos económicos, en el estrato I se puede observar que la actividad no es viable, lo que indica que los factores de producción no son remunerados adecuadamente. En el estrato dos y tres, en términos económicos, es viable la actividad ya que remunera costos fijos, costos variables, la mano de obra del productor, el costo de la inversión y la gestión empresarial, así como la depreciación.

Cuadro 6: Costos, ingresos y rentabilidad (\$)

\begin{tabular}{llrrr}
\hline & & Económico & Financiero & Desembolsado \\
\hline \multirow{3}{*}{ Estrato I } & $46,783.63$ & $30,713.07$ & $25,713.07$ \\
& Costos totales & $44,096.67$ & $44,096.67$ & $44,096.67$ \\
& Ingresos totales & $2,686.96$ & $13,383.60$ & $18,383.60$ \\
& Utilidad neta & -5.7 & 43.6 & 71.5 \\
\hline \multirow{3}{*}{ Estrato II } & Coeficiente de rentabilidad, \% & $137,990.83$ & $91,837.33$ & $79,737.33$ \\
& Costos totales & $148,275.20$ & $148,275.20$ & $148,275.20$ \\
& Ingresos totales & $10,284.37$ & $56,437.87$ & $68,537.87$ \\
& Utilidad neta & 7.5 & 61.5 & 86.0 \\
\hline \multirow{5}{*}{ Estrato III } & Coeficiente de rentabilidad, \% & $321,303.37$ & $244,385.87$ & $212,085.87$ \\
& Costos totales & $483,711.50$ & $483,711.50$ & $483,711.50$ \\
& Ingresos totales & $162,408.13$ & $239,325.63$ & $271,625.63$ \\
& Utilidad neta & 50.5 & 97.9 & 128.1 \\
\hline & Coeficiente de rentabilidad, \% & \multicolumn{2}{c}{. } &
\end{tabular}


Los precios de la miel se diferencian por el tipo de néctar que existe en la región. En este caso, la mayor producción es proveniente de la flor de mezquite ${ }^{(16)}$, que está entre las mejores cotizadas a nivel internacional ${ }^{(17)}$. Se identificó una relación inversa entre el volumen de producción y el precio respectivo, esto debido a que el productor del estrato I vende su producto al mercado local, mientras que los estratos II y III venden al mayoreo a un precio inferior en el mercado nacional e internacional (Cuadro 7). A pesar de que el pequeño apicultor alcanza un precio más alto, obtiene menor rentabilidad debido a factores como son los canales de comercialización, manejo técnico, economía de escala, valor agregado y limitados apoyos gubernamentales.

Cuadro 7: Mercados de la miel de abeja (\%)

\begin{tabular}{llll}
\hline & Local & Nacional & Internacional \\
\hline Estrato I & 92.78 & 7.22 & 0.00 \\
Estrato II & 34.50 & 23.00 & 43.50 \\
Estrato III & 24.38 & 27.50 & 47.12 \\
\hline
\end{tabular}

Fuente: Elaboración propia con datos de la encuesta a apicultores, 2018

\section{Precio de equilibrio}

El punto de equilibrio indica la magnitud de la producción y el precio al que se requiere vender o producir para evitar una perdida (Cuadro 8).

Cuadro 8: Precios de equilibrio por estrato (\$)

\begin{tabular}{lrrc}
\hline & Económico & Financiero & Desembolsado \\
\hline Estrato I & 69.95 & 45.92 & 38.45 \\
Estrato II & 47.39 & 31.54 & 27.38 \\
Estrato III & 33.51 & 25.49 & 22.12 \\
\hline
\end{tabular}

Fuente: Elaboración propia con datos de la encuesta a apicultores, 2018.

En el estrato I, el precio de $\$ 69.95$, es el necesario para cubrir el costo de todos los recursos, incluyendo la mano de obra familiar de la unidad de producción, gestión empresarial y costos de capital neto invertido. Los precios por arriba de $\$ 69.95$ generan un retorno al riesgo asumido por el productor; por debajo de esta cantidad implican retorno a la mano de obra del productor, gestión empresarial y retorno al capital neto invertido, menor al que se podría generar en el mejor uso alternativo de los recursos.

Siguiendo con el mismo estrato, el precio de equilibrio de $\$ 45.92$, es el precio necesario para cubrir los costos financieros de acuerdo con los sistemas contables, implica cero retribuciones a la mano de obra del productor. Los precios por debajo del precio de equilibrio implican una disminución de las ganancias retenidas. Un precio de $\$ 38.45$ cubre los gastos en efectivo del 
proceso productivo. Para el estrato II y III, los precios de equilibrio económico, financiero y desembolsado son más bajos respecto al primer estrato.

\section{Discusión}

Con base en la información recabada de la zona de estudio, se constata que la necesidad de adquirir los equipos de trabajo (aumento de capacidad del extractor, banco desoperculador, mini-spinner, recuperador de cera, tanque de sedimentación) y el material de campo (alzas, cámaras de cría, porta núcleos, etc.) se incrementa a medida que el apicultor adquiere más colmenas. Así los apicultores del estrato II y III que operan con más de 51 colmenas tienen una producción mayor a una tonelada y media, lo que conlleva a optar por aumentar la capacidad de instalación de maquinaria (acero inoxidable) favoreciendo la inocuidad de los productos. Lo encontrado, concuerda con estudios ${ }^{(18)}$ donde mencionan que el porcentaje de implementos aumenta de forma directa con el número de colmenas. Asimismo, en otra investigación similar, se afirma que el inventario o posesión de equipos complementarios, como extractor, banco desoperculador, embudo, cuchillos, entre otros, presentan un aumento progresivo con el tamaño del apiario ${ }^{(19)}$. Del mismo modo, otros autores ${ }^{(20)}$ llegan a la conclusión que a mayor número de colmenas mayor es su inversión.

A medida que los apicultores aumentan sus colmenas, la inversión en material de campo va superando a la inversión en maquinaria. Esto concuerda con lo reportado para el estado de Morelos, México ${ }^{(13)}$, donde se menciona que para el caso de la actividad apícola, las inversiones en maquinaria y equipo son mínimas, por ello las colmenas representan la mayor inversión en términos absolutos.

De acuerdo con los resultados de la región de estudio, en el primer estrato, la inversión por colmena fue de $\$ 933.33$ (coeficiente de variación del $20 \%$ ), \$819.98 (coeficiente de variación=23.7 \%) para el segundo y $\$ 768.51$ (coeficiente de variación $32 \%$ ) para el tercer estrato, con un promedio de $\$ 830.60$; lo cual es menor a lo que se reportó en las comunidades mayas del litoral centro de Yucatán, donde el promedio general de inversión por colmena fue de $1,201.3 \operatorname{pesos}^{(19)}$.

En lo que se refiere a la estructura de los costos, en general para México ${ }^{(9)}$ se estima que los costos de producción de la actividad apícola están compuestos mayormente por el costo variable, con una participación relativa de $67.1 \%$, mientras que los costos fijos representan una proporción del $32.9 \%$ respecto al costo total. Por otro lado, respecto al costo total, en Yucatán se reportó una contribución del $77.9 \%$ para el costo variable y $22.1 \%$ para el costo fijo $^{(19)}$. Con respecto al desglose de los costos financieros mencionados previamente, el promedio estimado a nivel nacional y estatal fue menor a lo estimado en Aguascalientes. De 
esta manera, en la zona de estudio, para el estrato I el costo variable financiero contribuyó con el $79.0 \%$ respecto al total, para el estrato II fue $81.3 \%$ y para el estrato III le correspondió el $79.2 \%$.

Para el caso específico de los costos variables, el azúcar representó el $53.0 \%$ de los costos variables totales para el primer estrato, $54.4 \%$ para el segundo estrato y $60.0 \%$ para el tercer estrato, con un promedio total de $55.8 \%$. Dichos porcentajes estimados presentan una diferencia significativa comparado con los resultados de otras investigaciones ${ }^{(19)}$ que reportan $38.7 \%$ en promedio. Una probable causa de esta diferencia porcentual, puede ser debido a que los intervalos entre una floración y otra son mas largos en la zona estudiada (dada la condición climática); por lo que el costo de alimentación se incrementa.

Por otro lado, para el estado de Nayarit hallaron, a través de un modelo para la generación de costos en empresas apícolas, que los productores con menores colmenas (estrato I) tienen una mayor erogación en el transporte, para el segundo (estrato II) el mayor gasto lo constituye el material de producción y para el tercer estrato es la mano de obra ${ }^{(21)}$.

Respecto al caso particular de los costos fijos, la depreciación (depreciación en infraestructura, equipos de protección y equipo) representó el $63.0 \%$ respecto al total de costos fijos. Lo anterior, fue más bajo a lo referido por otros autores ${ }^{(19)}$ donde representó $88.4 \%$ en promedio. Cuando se hace el análisis agregando los costos de oportunidad (análisis económico), existe una disminución en los rubros de costo fijo y costo variable.

Cuando la actividad apícola es la principal actividad económica, se manifiesta con la posesión de un tamaño mayor de apiarios, en contraste cuando se tienen pocas colmenas, los apicultores diversifican las actividades económicas ${ }^{(20)}$. Es por ello, que en los estratos II y III del presente trabajo fue mayor el número de productores que depende primordialmente de la apicultura.

También se encontró que se tiene una escasa diversificación en la producción de la colmena, el $90.7 \%$ produce sólo miel, siendo ésta su mayor fuente de ingreso. Esto coincide a lo observado en Argentina, que registraron un $82 \%$ en promedio ${ }^{(21)}$, mientras que en algunas regiones de México señalan que es el $99.5 \%{ }^{(19)}$.

Los precios de equilibro a nivel financiero estimados (para el primer estrato de $\$ 45.92$ por kilogramo de miel, \$31.54 por kilogramo de miel para el segundo y \$ 25.46 para el último) fueron similares a los reportados para algunas regiones de México ${ }^{(22)}$. En Nayarit se encontró que el punto de equilibrio para el apicultor que tiene 100 colmenas es de $\$ 14,865.00$ por ingresos, $\$ 73,715.00$ para el que tiene 450 colmenas y $\$ 52,642.00$ para el apicultor con 600 colmenas $^{(23)}$. 


\section{Conclusiones e implicaciones}

Se observó que la rentabilidad económica para los pequeños productores es negativa, mientras que para el segundo y tercer estrato son positivos. La rentabilidad positiva de los medianos y grandes productores puede deberse a la escala de producción, ya que disminuyen los costos de los insumos al tener volúmenes de compra altos y precio de venta asegurados. De esta manera, con base en los resultados expuestos, se puede deducir que, en el estado de Aguascalientes, el estrato II y III practican la apicultura a mediana y alta escala; mientras que el estrato I, es una apicultura de baja escala, caracterizado por un manejo tradicional, donde no contemplan los costos administrativos. Para la consolidación de los pequeños productores, se requiere la inversión de capital y tecnología para la formación de la pequeña empresa, con el objetivo de generar mayor producción y poder negociar en el mercado. De acuerdo con los datos analizados, se puede concluir que en la entidad existe un potencial de diversificación de los subproductos de gran demanda en el mercado como la jalea real, propóleo, polen, cera, núcleos de abejas y reinas.

\section{Literatura citada:}

1. SIAVI. Sistema de Información Comercial Vía Internet. http://www.economiasnci.gob.mx/. Consultado Sep 12, 2019.

2. García GM, Ríos OLA, Álvarez-Castillo J. La polinización en los sistemas de producción agrícola: Revisión sistemática de la literatura. Idesia, 2016;34(3):53-68.

3. SIAP. Sistema de Información Agropecuaria y Pesquera. Produccion Pecuaria. Resumen nacional. https://www.gob.mx/siap/acciones-y-programas/produccion-pecuaria. Consultado 20 Oct, 2019.

4. SIACON-NG. Sistema de Información Agroalimentaria de Consulta. Consultado 20 Oct, 2019.

5. Rodriguez S. Gana miel de aguascalientes primer certificado TIF. NW aguascalientes. (12 de febrero de 2018). Recuperado de https://newsweekespanol.com/2018/02/gana-mielde-aguascalientes-primer-certificado-tif/

6. Medina CSE, Álvarez JM, Portillo VM, Terrazas GGH. Influencia de los factores ambientales y de manejo en la segunda temporada de producción de miel de abeja en Aguascalientes, México. Rev Esp Estud Agrosoc Pesq 2014;(238):65-80. 
7. Martínez BHA, Hernández AEG. Análisis de brechas tecnológicas e identificación de oportunidades de vinculación con organizaciones y empresas del sector apícola en Aguascalientes.. 1ra ed. (versión electrónica). Aguascalientes, México. Universidad Autónoma de Aguascalientes; 2017.

8. Castellanos PBP, Gallardo LF, Sol SA, Landeros SC, Diaz PG, Sierra FP, Santibañez JL. Impacto potencial del cambio climático en la apicultura. Rev Iberoam Bioecon Cambio Clim 2016;2(1):1-19.

9. Magaña MA, Leyva CE. Costos y rentabilidad del proceso de producción apícola en México. Contad Adm 2011;(235):99-119.

10. Franco VH, Siqueiros ME, Hernández EG. Flora apícola del estado de aguascalientes. 1ra ed. México. Universidad Autónoma de Aguascalientes; 2012.

11. Sagarnaga VLM, Salas GJM, Aguilar AJ. Ingresos y costos de produccion. Unidades representativas de produccion. Trópico humedo y mesa central. Centro de Investigaciones Economicas, Sociales y Tecnologicas de la Agroindustria y la Agricultura mundial (CIESTAAM) 2014;1 (1): 19-47.

12. Instituto Nacional de Estadística y Geografía (México). Anuario estadístico y geográfico de Aguascalientes: INEGI; 2017.

13. Vélez IA, Espinosa GJA, Amaro GR, Arechavaleta VME. Tipología y caracterización de apicultores del Estado de Morelos, México. Rev Mex Cienc Pecu 2016;7(4):507-524.

14. Fachini C, Firetti R, Cardoso-Oliveira E, Assiz-Caravalo A. Perfil da apicultura em Capão Bonito, Estado de São Paulo: aplicação da análise multivariada. Rev Econom Agríc 2010;57(1):51-63.

15. Baca UG. Evaluación de proyectos. 7a ed. México. McGraw-Hill/Interamericana Editores, SA de CV; 2013.

16. Medina CSE, Tirado GDN, Portillo VM, López SMA, Franco OVH. Environmental implications for the production of honey from mesquite (Prosopis laevigata) in semiarid ecosystems. J Apic Res 2018;57(4):507-515.

17. Soto MLE, Elizarra BR, Soto MI. Situación apícola en México y perspectiva de la producción de miel en el Estado de Veracruz. Rev Estrateg Desarro Empresarial 2017;3(7):40-64.

18. Contreras-Uc LC, Magaña MA, Sanginés JR. Características técnicas y socioeconómicas de la apicultura en comunidades mayas del Litoral Centro de Yucatán. Acta Univ 2018;28(1):44-86. 
19. Contreras- Uc LC, Magaña MA. Costos y rentabilidad de la apicultura a pequeña escala en comunidades mayas del Litoral Centro de Yucatán, México. Invest cien 2017;25(71):52-58.

20. Contreras EF, Perez AB, Echazarreta CM, Cavazos AJ, Macias JO, Tapia GJM. Características y situación actual de la apicultura en las regiones Sur y Sureste de Jalisco, Mexico. Rev Mex Cienc Pecu 2013;4(3):387-398.

21. Ulmer J, Travadelo M, Caporgno J, Castignani H. Caracterización de los modelos de producción apícola representativos de la zona central de la provincia de Santa Fé. Cienc Agron 2011;(XVIII):043-049.

22. Dolores MG, Santiago MDJ, Arana CJJ, Utrera QF. Estudio del impacto de la actividad apícola en el Istmo de Tehuantepec, Oaxaca, México. Agric Soc Desarro 2017;14(2):187-203.

23. Ulloa CRR, Anzalo VJE, Martínez VM, Martínez GS, Lenin OJL. Generacion de un modelo para la determinación de costos de empresas productoras de miel en el estado de Nayarit. Rev Mex Agroneg 2014;35(2014):1072-1081. 